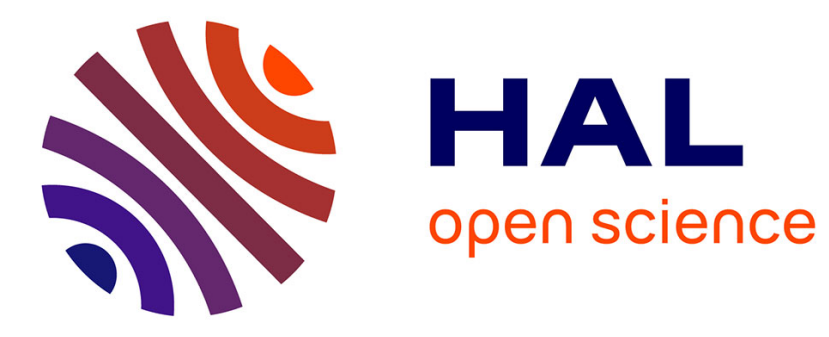

\title{
Mobilité des puits quantiques étroits
}

Gérald Bastard, C. Delalande, Y. Guldner, M. Voos

\section{To cite this version:}

Gérald Bastard, C. Delalande, Y. Guldner, M. Voos. Mobilité des puits quantiques étroits. Revue de Physique Appliquée, 1989, 24 (1), pp.79-81. 10.1051/rphysap:0198900240107900 . jpa-00246032

\section{HAL Id: jpa-00246032 https://hal.science/jpa-00246032}

Submitted on 1 Jan 1989

HAL is a multi-disciplinary open access archive for the deposit and dissemination of scientific research documents, whether they are published or not. The documents may come from teaching and research institutions in France or abroad, or from public or private research centers.
L'archive ouverte pluridisciplinaire HAL, est destinée au dépôt et à la diffusion de documents scientifiques de niveau recherche, publiés ou non, émanant des établissements d'enseignement et de recherche français ou étrangers, des laboratoires publics ou privés. 
Classification

Physics Abstracts

$73.20-73.40 \mathrm{~L}$

\title{
Mobilité des puits quantiques étroits
}

\author{
G. Bastard, C. Delalande, Y. Guldner et M. Voos \\ Groupe de Physique des Solides de l'ENS, 24 rue Lhomond, 75005 Paris, France
}

(Reçu le $1^{e r}$ juillet 1988, révisé le 8 septembre 1988, accepté le 7 octobre 1988)

\begin{abstract}
Résumé. - Nous présentons les résultats du calcul de la mobilité ohmique à basse température pour des puits quantiques étroits.
\end{abstract}

Abstract. - We report on low temperature ohmic mobility calculations in narrow quantum wells.

De par leur capacité à stocker plus de charges que les simples hétérojonctions les puits quantiques à modulation de dopage peuvent être intéressants pour la réalisation de TEGFET. Il est par ailleurs connu que la mobilité des gaz d'électrons quasi 2D des puits quantiques est notablement plus faible (à concentration 2D $n_{\mathrm{e}}$ fixée) que celle des simples hétérojonctions. La nécessaire présence d'une interface « inverse » (c'est-à-dire correspondant à l'épitaxie de $\mathrm{GaAs}$ sur $\mathrm{Ga}(\mathrm{Al}) \mathrm{As}$ ) dans un puits quantique peut être la raison d'un tel phénomène ; soit que l'interface inverse soit très rugueuse, soit qu'elle conduise à la ségrégation des impuretés résiduelles (carbone? oxygène ?).

Nous avons examiné l'effet de la rugosité d'interface $[1,2]$, d'impuretés chargées situées à l'interface d'un puits quantique et du désordre d'alliage (lorsqu'il affecte le matériau puits) sur la mobilité basse température de puits quantiques étroits. Par étroits on entend que la quantification en $z$ soit très marquée et que seule la sous-bande $\mathrm{E}_{1}$ soit peuplée (limite électrique quantique). Les niveaux d'énergie et fonctions d'onde ont été calculés à l'approximation de Hartree en traitant la courbure de bande en perturbation, le puits étant supposé dopé symétriquement par modulation. La mobilité a ensuite été évaluée par les techniques usuelles à l'approximation de Born à $T=0 \mathrm{~K}$, en tenant compte de l'écrantage des potentiels par les électrons quasi $2 \mathrm{D}$ et en incluant les effets d'extension finie de la fonction d'onde le long de l'axe de croissance. Le potentiel créé par les défauts d'interface est pris comme une somme de boîtes à chapeaux gaussiennes $\left(\exp \left(-\rho^{2} / a^{2}\right)\right)$ dans le plan des couches et de profondeur $h$ le long de l'axe de croissance. On obtient un résultat identique à celui du modèle des fonctions de corrélations gaussiennes des défauts

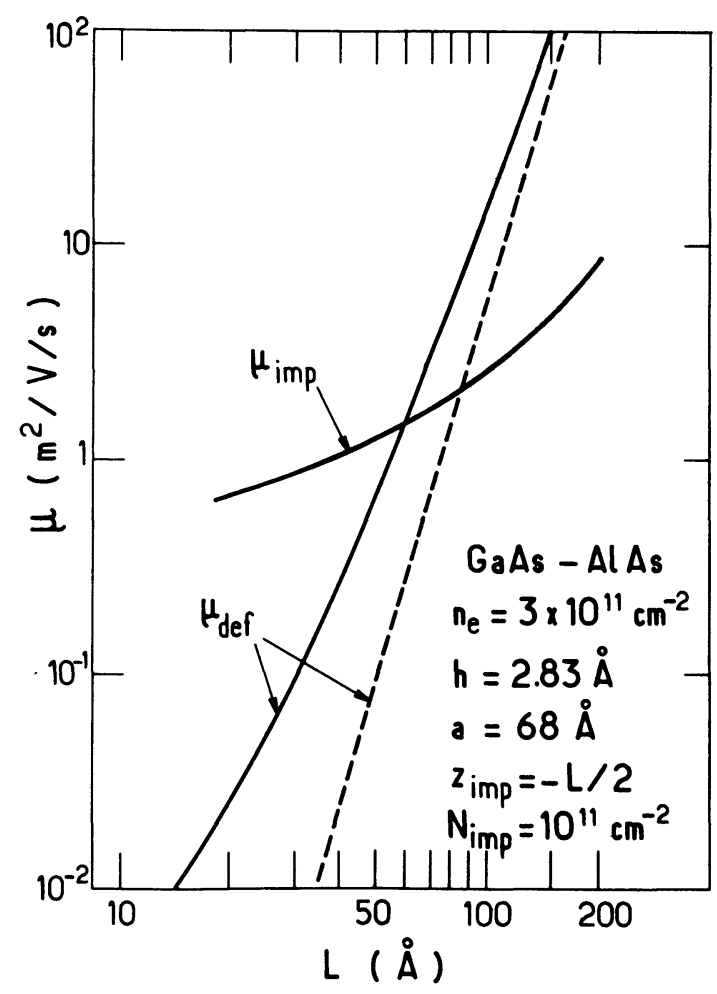

Fig. 1. - Mobilité $\mu$ fonction de l'épaisseur du puits $L$ pour le système GaAs-AlAs. Les traits tiretés correspondent à l'hypothèse d'une hauteur de barrière infinie.

[The low temperature mobility of GaAs-AlAs single quantum wells is plotted against the well thickness $L$. The dashed line corresponds to the assumption of an infinite barrier height.] 
d'interface $[1,2]$ pourvu que les défauts gaussiens soient en nombre $N_{\mathrm{d}}=2 / \pi a^{2}$ par unité de surface. Les valeurs $h=2,83 \AA$ et $a=68 \AA$ ont été choisies pour les applications numériques, en conformité avec les références $[1,2]$.

Les figures 1 et 2 présentent les résultats obtenus pour le système $\mathrm{GaAs}-\mathrm{Ga}_{1-x} \mathrm{Al}_{x} \mathrm{As}(x=0,3$ et 1$)$ en fonction de l'épaisseur du puits $L$. On constate que la diffusion par les défauts d'interface n'est effective que pour les puits minces $(L \leqslant 60 \AA)$. Les traits tiretés des figures 1 et 2 correspondent à l'hypothèse $V_{\mathrm{b}}=\infty$ et conduisent à une loi $\mu \approx L^{6}$. On se rend compte que cette loi n'est suivie que pour des épaisseurs telles que les défauts d'interface ne constituent plus un mécanisme de diffusion efficace. On constate également que $\mu_{\text {imp }}$ varie plus faiblement avec $L$. Cela tient à ce que le potentiel coulombien, même écranté, est à plus long rayon d'action que les défauts d'interface qui, pour diffuser efficacement, requièrent une densité de probabilité de présence des porteurs importante à l'interface.

La figure 3 présente la variation du temps de relaxation $\tau$ de la vitesse $\left(\tau=m^{*} \mu / e\right)$ en fonction de l'épaisseur du puits pour le système InP$\mathrm{Ga}_{0,47} \mathrm{In}_{0,53}$ As. Le désordre d'alliage a été inclus dans les calculs car c'est un mécanisme de diffusion important dans ces structures. On note que $\tau_{\text {alloy }}$ présente un minimum en fonction de $L$. En deçà de

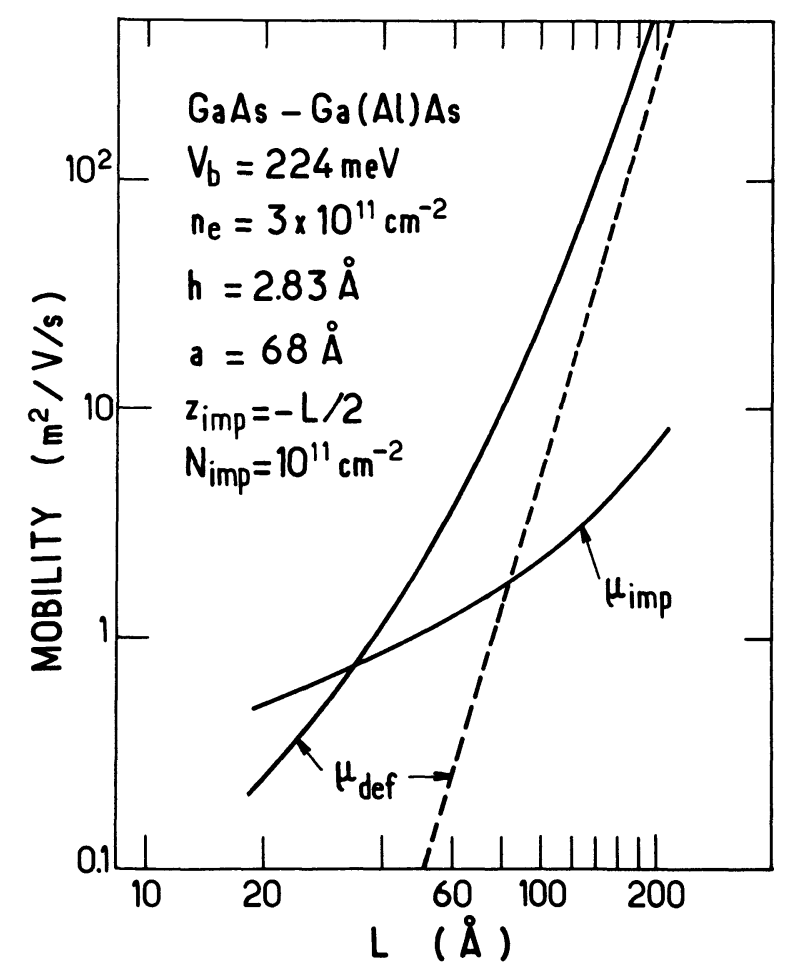

Fig. 2. - Ibidem figure 1 mais pour le système GaAs$\mathrm{Ga}_{0,7} \mathrm{Al}_{0,3}$ As.

[Same as figure 1 but for GaAs- $\mathrm{Ga}_{0.7} \mathrm{Al}_{0.3} \mathrm{As}$ quantum wells $\left(V_{\mathrm{b}}=224 \mathrm{meV}\right)$.]

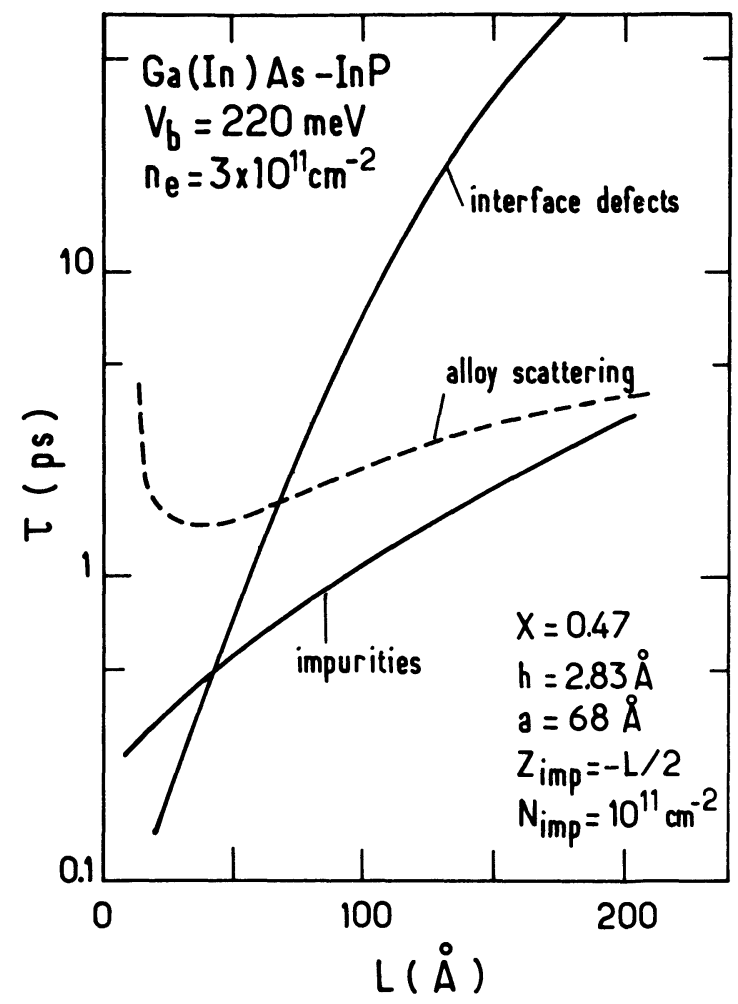

Fig. 3. - Le temps de relaxation $\tau$ de la vitesse est porté en fonction de l'épaisseur du puits $L$ pour le système $\mathrm{Ga}_{0,47} \mathrm{In}_{0,53}$ As-InP.

[The low temperature velocity relaxation time of $\mathrm{Ga}_{0.47} \mathrm{In}_{0.53}$ As-InP single quantum wells is plotted against the well thickness $L$.]

ce minimum la délocalisation progressive de la fonction d'onde dans la barrière (InP) rend le désordre d'alliage moins efficace. De même, aux grands $L$, tant que la limite électrique quantique demeure justifiée, $\tau_{\text {alloy }} \approx L$ : la fonction d'onde de l'état fondamental se délocalise sur tout le puits et les porteurs sont de moins en moins affectés par des phénomènes se produisant sur une échelle de longueur fixe (la cellule élémentaire).

Finalement, la figure 4 établit une comparaison entre les dépendances en $L$ des temps de relaxation de la vitesse $\left(\tau_{\mathrm{s}}\right)$ et de vie des niveaux $\left(\tau_{\mathrm{k}}\right)$ au niveau de Fermi pour des électrons quasi 2D dans des puits $\mathrm{GaAs}-\mathrm{Ga}_{0,7} \mathrm{Al}_{0,3} \mathrm{As}$. Seules les diffusions par les défauts d'interface et par des impuretés à l'interface ont été prises en compte. On constate que $\tau_{\mathrm{s}}$ et $\tau_{\mathrm{k}}$ sont pratiquement identiques pour la diffusion par les défauts d'interface tandis que $\tau_{k}<\tau_{s}$ pour la diffusion par les impuretés ionisées. Une fois encore c'est la porté spatiale des deux types de diffuseurs qui explique cette différence. $\tau_{\mathrm{s}}=\tau_{\mathrm{k}}$ pour les potentiels à court rayon d'action (diffuseurs isotropes); tandis que la plus longue portée des potentiels coulombiens écrantés, qui privilégie la 


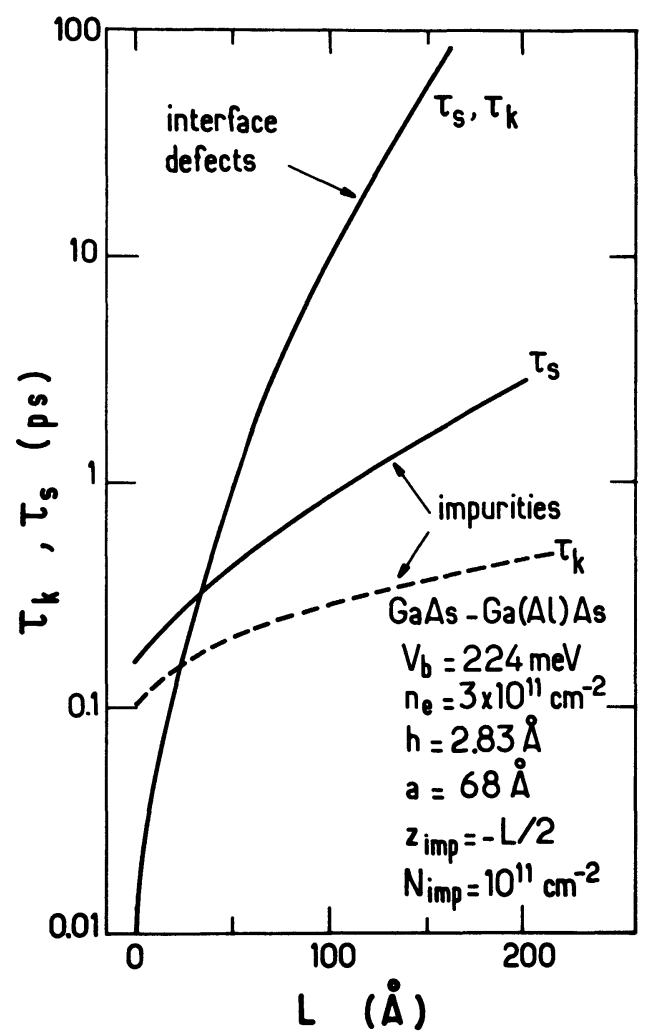

Fig. 4. - Comparaison des dépendances des temps de relaxation de la vitesse $\left(\tau_{\mathrm{s}}\right)$ et des niveaux $\left(\tau_{\mathrm{k}}\right)$ avec l'épaisseur $L$ de puits quantiques $\mathrm{GaAs}-\mathrm{Ga}_{0,7} \mathrm{Al}_{0,3} \mathrm{As}$.

[The low temperature velocity relaxation time $\left(\tau_{\mathrm{s}}\right)$ and level lifetime $\left(\tau_{\mathrm{k}}\right)$ of electrons in $\mathrm{GaAs}-\mathrm{Ga}(\mathrm{Al}) \mathrm{As}$ single quantum wells are plotted versus the quantum well thickness $L$.]

diffusion aux petits angles, accroît le rapport $\tau_{s} / \tau_{k}$. Ceci montre que le temps de relaxation que l'on peut tirer d'une mesure de mobilité n'est pas forcément celui qui contrôle l'élargissement des niveaux.

\section{Remerciements.}

Ce travail a été en partie subventionné par la DRET (contrat 87 1407). Le GPS de l'ENS est Unité Associée (UA 17) au CNRS.

\section{Bibliographie}

[1] SaKaki, H., Noda, T., Hirakawa, K., TanaKa, M. et Matsusue, T., Appl. Phys. Lett. 51 (1987) 1934.
[2] Gottinger, R., Gold, A., Abstreiter, G., WeIMANN, G. et ScHLAPP, W., Europhys. Lett. 6 (1988) 183. 